\title{
Scar Resurfacing
}

Fiona M. Wood

\section{Contents}

$36.1 \quad$ Overview -312

36.2 The Mechanism of the Initial Skin Injury and Clinical Pathway of Healing - 312

36.3 The Timing of the Intervention - 313

36.4 The Techniques for Preparing the Scar Wound Bed for Resurfacing - 313

36.5 The Techniques of Wound Repair for Resurfacing - 314

36.6 Post-Intervention Scar Management - 315

36.7 Conclusion $-\mathbf{3 1 5}$

References -316 


\subsection{Overview}

Established scarring can have an impact on the function and aesthetics of the skin related to the volume of the fibrous tissue, the vascularity of the area, the colour mismatch with the adjacent normal skin and disruption of the skin adnexal structures. Resurfacing is a technique which involves the disruption of the epidermal surface of a scarred area with removal of a varying volume of the underlying scarred dermal element, followed by a controlled repair of the surface.

The aim of resurfacing is to improve the character of the scarred area with a resulting scar more closely matching the surrounding uninjured skin. This can be focused on improving the texture of the surface, the pigment match or reduction of the volume of scar. Resurfacing will not address scar contracture or the absence of skin adnexal structures, with the exception of full-thickness excision and reconstruction with fullthickness skin graft or flap repair. The character of the skin varies across body sites such that the specialist nature of the skin site must be considered when planning any intervention. A complex scar reconstruction may be undertaken as a combined procedure with strategies designed to address the range of issues including resurfacing.

The timing and technique of the procedure are guided by the preceding clinical situation and specifically by the understanding of the conditions at the time of wound healing and subsequent scar development [1]. It is essential to understand that the opportunity to improve the scar depends upon the opportunity to better control the wound healing at the time of the resurfacing intervention.

There are a number of considerations to be taken into account when planning the interventions to improve the scar quality by control of the secondary wound healing:

- The mechanism of the initial skin injury and clinical pathway of healing, with specific reference to the initial techniques of wound repair, the time to healing and the presence of infection and other comorbidities influencing wound healing

- The time from the initial insult, considering if the scar is fully mature or there are symptoms of the scar, or epithelial stability compromise, such that early intervention is being driven in an immature active scar

- The techniques available for the intervention in terms of the scar intervention and subsequent wound repair and post-intervention scar management

- Counselling that it is possible to improve the scar but not to eradicate the scar with the current stage of knowledge of wound healing
For the scar resurfacing intervention to translate into an improved scar outcome, it is important to understand the clinical opportunities which offer an improvement on the initial treatments which have resulted in the unacceptable scar. For example, if the plan is to repeat the same procedure, such as a surgical shave and split-thickness skin graft, then there has to be a significant difference in the clinical situation to advise that such a strategy offers the opportunity of scar reduction. This may be appropriate as in a mature scar there is control over the timing of the intervention such that it is not associated with the inflammation of the acute episode. Alternatively, there may be the use of a new method of scar reduction such as laser or a method to reduce the time to healing such as a cellbased therapies.

The chapter aims to outline strategies for scar improvement by disruption of the scar surface and control of the secondary healing process. In the context of this discussion, it is essential to articulate clearly the potential gains on a risk matrix that is realistic for the patient. The more severe a scar is, the greater the potential for improvement, as compared to a good-quality scar when the risk of worsening the scar may sensibly limit the interventions.

\subsection{The Mechanism of the Initial Skin Injury and Clinical Pathway of Healing}

It is well known that scar outcome is related to the mechanism of injury such that burn injury is frequently associated with an aggressive scar outcome. The surface area of the body involved in the injury is also a driver of the outcome with the challenges in healing large surface areas in a timely fashion. Further, the body site of the injury is also influential with areas such as the presternal and deltoid regions and areas of skin tension being associated with poor scar outcome.

The time to healing is a key element in the scarring process and can be affected by local and [1] systemic factors, infection, necrotic tissue, haematoma and comorbidities such as diabetes, nutritional deficiency, drugs such as steroids and retinoid in acne treatment. The clinical history is key in understanding what can be optimised to improve the quality of healing and what factors may have been influential in the scar quality. Resurfacing is an elective procedure, and therefore, the preoperative optimisation of the patient's condition is possible and needs attention. The history of infection is of importance in ensuring the intervention is covered by the appropriate antibiotics or the risk of triggering secondary problems such as herpes is considered with the appropriate prophylaxis. 
The knowledge of the initial intervention will also assist in the understanding of how resurfacing can be used in the post-acute phase to rehabilitate the scarred area [2]. Sharp dissection and haemostasis followed by careful approximation of the opposing surfaces with a suture designed to minimise inflammation are setting up the healing for the best outcome possible. In a wound such as a burn requiring cover of surface area, matching the repair to the defect in depth and body site with a focus on time to healing will again set up the best possible outcome. The concept of scar minimisation by meticulous attention to detail at the time of the initial intervention is covered in detail in $>$ Chap. 23.

Despite the best efforts, the post-intervention period has influence on the outcome with infection control and techniques of wound support and post-healing scar management having an ongoing role.

The knowledge of the clinical pathway, both initially and the proposed interventions, is essential when considering the following question:

- Is it possible to re-wound the scar and control the healing in such a way as to significantly reduce the risk of scar recurrence?

\subsection{The Timing of the Intervention}

The natural history of scarring is such that in the majority of cases, the scar severity will plateau between 6 and 12 weeks with subsequent remodelling leading to a mature scar construct by 52 weeks. However, the scar will continue to improve over the subsequent years. In order to have control over the extent of scar re-excision, it may be prudent to wait until the scar is mature prior to intervention. The scar may well improve over time to the point that it is acceptable. The progress towards maturation can be influenced by a range of non-invasive strategies highlighted in the chapters in section VIII. When considering resurfacing interventions, it is important to consider what aspects of the scar are of concern and how this could be influenced by time and conservative strategies.

However, there may be indications such as unstable epithelial surface, unsightly mismatched skin surfaces, or symptoms related to the scar such as itching which drives the decision for earlier intervention.

On the other hand, concern that a scar may be too old to consider treatment is unfounded, with improvement in the quality being achieved after many years.

Timing is an element in the risk matrix that needs to be considered in the context of the patient's symptoms and concerns. Early intervention may be explored related to specific techniques such as the re-suture a scar mismatch in height or laser to reduce pruritus, whereas in a florid vascular scar, more aggressive early intervention may add to the overall scarring pruritus, whereas in a florid vascular scar, more aggressive early intervention may add to the overall scarring [3]. The discussion with the patient needs to include the options such as time with the risks and benefits. It is useful to have a scar assessment tool which assists in understanding the character of a scar and facilitates the discussion and clinical decision-making.

The assessment of the scar can be done in a number of ways, e.g. surface scanning focusing on specific aspects such as stiffness or colour [4], subjective standard assessment systems, validated questions [5] and clinical photography. When embarking on scar revision, the documentation of the scars is essential in tracking progress over time; the options are explored in detail in section VII.

\subsection{The Techniques for Preparing the Scar Wound Bed for Resurfacing}

The prerequisite to prepare the scar for resurfacing is the removal or disruption of the epithelial layer by techniques including chemical, laser, dermabrasion and sharp dissection [6].

Chemical peels have been widely used aiming to remove the surface epithelium to a limited depth such that rapid healing by secondary intention is achieved with limited improved scar outcome.

There has been an expansion of the laser techniques used to remove or to disrupt the epithelial surface as explored in the chapters in section IX. Again, laser in isolation can be used with surface damage limited to a superficial level such that the healing by secondary intention is rapidly achieved. The use of fractional laser with discrete microthermal zones has been shown to initiate scar remodelling within the dermis. Further, the ablative lasers can be a tool used to prepare the wound bed for secondary interventions as described below.

Dermabrasion can be achieved with manually using abrasive sand papers, or mechanically with rotating brush or burr. The depth of tissue removal can be controlled, and the contour can be guided using adjuncts such as injection or surface coating with agents such as methylene blue. Surface irrigation is necessary to mitigate against heat damage and to remove the debris from the surface.

The sharp dissection tools range from freehand scalpels to mechanised guarded blades allowing serial shaving of the scar surface. Again, the depth of the tissue excision is dictated by the strategy of consequent wound healing [7]. In situations of superficial shave, the aim may be controlled healing by secondary intention. However, the use of sharp dissection lends itself to de- 
bulking of the scar volume such that the wound bed does not retain the capacity to heal within a timeframe that would limit the secondary scar risk [8].

The extent of the excision of the fibrotic dermal layer can range from total scar excision to minimal removal of the tissue. With the retention of the scarred bed, the overall scar result relies on the remodelling, driven by the interaction of the cells of the new epidermal layer and those in the retained scar construct.

\subsection{The Techniques of Wound Repair for Resurfacing}

In the situation of total scar excision, a full-thickness skin replacement is the ideal reconstruction and is considered in $>$ Chap. 37, using flap repair, and $>$ Chap. 39 exploring the use of skin substitutes.

In some instances, an appropriate donor site for a full-thickness skin graft may allow full-thickness reconstruction, or the use of tissue expansion can facilitate local flap repair with the most similar skin characteristics. In complex scar reconstruction, the functional restriction may require the introduction of tissue from a remote uninjured body site using local or free tissue transfer explored in $>$ Chaps. 37 and 40.

In a scar which has been prepared using limited removal of the epidermal surface, healing by secondary intention may be appropriate. In these cases, specific attention to detail is required to protect the healing wound surface [9]. The key to limiting the risk of worsening the scar is the time to healing. There are many strategies employed to facilitate healing with advanced technology dressing systems, both synthetic and biologically based.

When the scar has been de-bulked, then secondary repair of the surface should be considered as prolonged healing by secondary intention will be associated with an increased scar risk. The use of thin split-thickness skin graft can result in an improvement of the scar in terms of contour and colour. However, this can be unpredictable with some split-thickness skin grafts associated with abnormal pigmentation. There is also the drawback of the donor site availability in terms of area and site specificity.

Matching the donor and recipient site is a key element in improving the potential outcome from scar resurfacing. For example, the use of full-thickness skin grafts to release scar contractures of the palm of the hand will frequently afford an excellent functional release but is associated with significant mismatch in terms of the skin characteristics. The ability to match donor site is most frequently limited by the surface area required. The donor site itself can be resurfaced such that a thick split-thickness or full-thickness harvested area is secondarily covered by a thin split-thickness skin graft aiming to reduce the overall donor site morbidity.

The use of tissue expansion techniques may also be used to address the donor site limitation. The concept of tissue expansion can be extended beyond the fullthickness skin construct, to the cells essential for reepithelisation. The development of the processes of cultured epithelial autograft was specifically to address the need of large surface area skin cover in major burn injuries. The techniques of laboratory-based tissue expansion can provide epithelial cells as sheets or suspension from a limited donor site which will retain the epithelial characteristics of the donor site. The drawback of such cell-based therapies is the reliance on the laboratory environment and the time taken to achieve the expansion via the tissue culture process.

The first steps of the cell-based expansion process have been incorporated into a medical device as a lab in a box, ReCell ${ }^{\mathrm{TM}}$. The technique of harvesting at the point of care of the cells of the dermal-epidermal junction, for delivery as a cellular suspension, provides a method with an expansion ratio of up to $1-80$. The technique uses the wound bed as the tissue culture environment with the cellular suspension adhering to the prepared wound surface and the cells migrating and differentiating into an intact epithelium. The use of the cell suspension from the dermal-epidermal junction allows the transfer of keratinocytes, melanocytes and papillary dermal fibroblasts in their normal ratio [10]. The use of such a cellular suspension in resurfacing is particularly useful in treating hypopigmentation. The melanocytes within the suspension will re-establish in the prepared wound, and re-pigmentation is seen over the subsequent 6-12 weeks. The introduction of the cell suspension facilitates the epithelial repair reducing the time to healing and therefore the risk of secondary scarring.

There is also interesting basic science evidence that the interaction between the keratinocytes and fibroblasts has influence on the phenotype of the fibroblasts impacting on collagen deposition [11].

The use of cell-based therapies is an opportunity to expand the surface area of wound cover possible for a given donor site with the appropriate cell phenotype. The seeding of the cells onto a de-bulked scarred wound bed reduces the time to achieve an intact epithelial surface with the potential to introduce melanocyte and keratinocyte characteristics to match the area. The introduction of an uninjured cell phenotype has a theo- 
retical impact on the scar remodelling as the scar matures.

Whatever method of scar resurfacing is used, the objective remains constant, to improve the area of scar such that the scar blends better into the surrounding skin.

\subsection{Post-Intervention Scar Management}

The wound healing is only the first phase in the process of scar remodelling post resurfacing, and attention to detail at all stages is essential. The post-intervention dressing systems may involve a range of products focused on providing an environment of healing with infection control and protection of the fragile wound surface.

Further, the protection of the healed epithelial surface and control of the environment are important as the dressings are removed and the surface exposed. At this stage, it may be prudent to consider the non-invasive conservative strategies for scar minimisation such as silicones and massage as explored in the chapters of section VIII. At all stages, hygiene is an essential element to prevent secondary infection and breakdown whilst the cell surface is fragile and immature.

For decades, pressure garments have been used in the attempt to control scar progression particularly post burn injury. The mechanism of action is debated, but it remains a widespread practise. The fabrics used vary in terms of the pressure delivery and the moisture wicking characteristics. A light pressure fabric can be used to protect the area in the early stages post healing if well designed to avoid trauma and sheering.

Sun protection is also important not only to prevent sunburn of course but also to avoid the overstimulation of the melanocytes with the risk of hyperpigmentation in the resurfaced area. Simple advice such as wearing a hat and topical sunblock needs to be considered as part of the post-intervention information given to the patient. The advice will need to be tailored to the patient in relation to their environment.

\subsection{Conclusion}

The clinical decision to undertake a scar resurfacing procedure needs to bring together the needs of the patient and the characteristics and history of the scar,

along with the tool box of potential interventions (- Table 36.1).

The choice of the specific resurfacing technique will then consider the triangle of care to specifically address the patient's needs in the context of the clinician's knowledge and experience along with the technology environment available (- Fig. 36.1). The diagnosis of the scar with the inclusion of an assessment tool will give clarity to the clinical discussion with the focus on the aspects of the scar causing the concern.

Understanding the natural history of the scarring process will help in giving advice to the patient, explaining the opportunities of conservative therapies and time to improve the scar. Then, choosing the technique that will give most benefit for least risk is the key to safely improving the scar. There are many ways of resurfacing, but understanding the need for bulk reduction, repigmentation or stabilising the epithelium of the scar will drive the clinical decision.

In the clinical counselling, being realistic that the scar may be reduced and blended to the surrounding skin rather than eradicated facilitates decision-making for the patient.

- Table 36.1 Understanding the clinical details of the scar and the impact of the symptoms on the patient to drive the most appropriate clinical intervention for the individual

a

Scar Assessment to guide intervention

Clinical history

- Mechanism of original scar process

- Timeframe of scar development

- Time to healing

- Technique used to achieve healing

- Presence of infection

- Systemic health and nutrition

b

Scar Resurfacing

Techniques to prepare the scar for resurfacing

- Sharp dissection

- Dermabrasion

- Laser techniques

- Chemical preparation
Scar symptoms

- Itching

- Pain

- Stiffness

- Dryness

- Epithelial instability

- Thickness

- Vascularity

- Colour

Techniques to control secondary wound healing

- Cell based therapies

- Advanced dressing systems

- Infection control

- Systemic optimisation 
- Fig. 36.1 The triangle of care bringing together essential considerations for clinical decision making
Scar Revision

What the patient needs

Characteristics of the scar

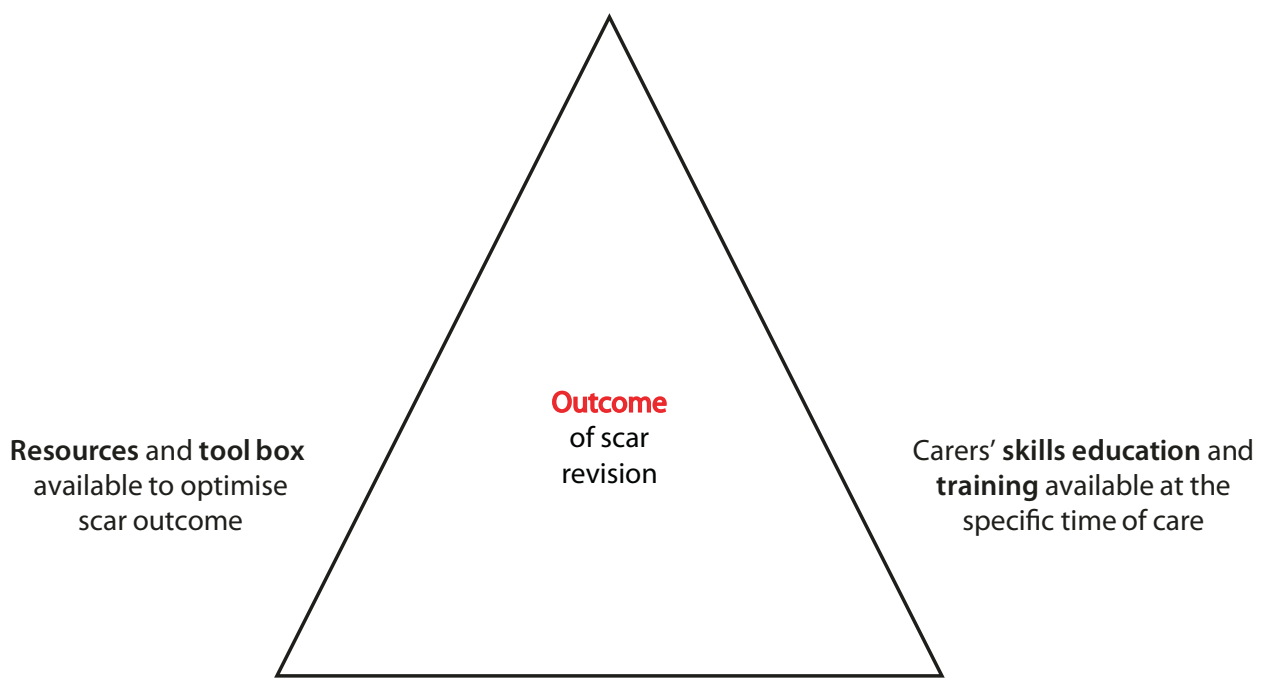

\section{- Take-Home Messages}

- Clinical history of the patient and the scar is pivotal in the decision-making process.

- Scar assessment is key in tracking the impact of the scar intervention over time.

- Be clear on what elements of the scar need to be treated.

- Focus the intervention on the outcome required.

- The time to healing is a key element driving scar outcome.

- Meticulous attention to detail needs to be continued to the time of scar maturity.

- Education of the patient along the clinical journey is essential for a successful outcome.

\section{References}

1. Finlay V, Burrows S, Burmaz M, Yawary H, Lee J, Edgar D, Wood F. Increased burn healing time is associated with higher Vancouver Scar Scale score. Scars Burns Heal. 2017;3: 2059513117696324
2. Hess C. Checklist for factors affecting wound healing. Adv Skin Wound Care. 2011;24(4):192.

3. Akaishi S, Ogawa R, Hyakusoku H. Keloid and hypertrophic scar: neurogenic inflammation hypotheses. Med Hypotheses. 2008;71(1):32-8.

4. Gankande TU, Duke JM, Danielsen PL, HM DJ, Wood FM, Wallace HJ. Reliability of scar assessments performed with an integrated skin testing device - the DermaLab Combo ${ }^{\circledR}$. Burns. 2014;40(8):1521-9.

5. Simons M, Price N, Kimble R, Tyack Z. Patient experiences of burn scars in adults and children and development of a healthrelated quality of life conceptual model: a qualitative study. Burns. 2016;42(3):620-32.

6. Pugliese E, Coentro JQ, Raghunath M, Zeugolis DI. Wound healing and scar wars. Adv Drug Deliv Rev. 2018;129:1-3.

7. Onur Erol O, Atabay K. The treatment of burn scar hypopigmentation and surface irregularity by dermabrasion and thin skin grafting. Plast Reconstr Surg. 1990;85(5):754-8.

8. Bradley DT, Park SS. Scar revision via resurfacing. Facial Plast Surg. 2001;17(04):253-62.

9. Tarijian A, Goldberg D. Fractional ablative laser skin resurfacing: a review. J Cosmet Laser Ther. 2011;13(6):262-4.

10. Wood FM, Giles N, Stevenson A, Rea S, Fear M. Characterisation of the cell suspension harvested from the dermal epidermal junction using a ReCell ${ }^{\circledR}$ kit. Burns. 2011;38(1):44-51.

11. Ghaffari A, Kilani RT, Ghahary A. Keratinocyte-conditioned media regulate collagen expression in dermal fibroblasts. J Investig Dermatol. 2009;129(2):340-7.

Open Access This chapter is licensed under the terms of the Creative Commons Attribution 4.0 International License (http://creativecommons. org/licenses/by/4.0/), which permits use, sharing, adaptation, distribution and reproduction in any medium or format, as long as you give appropriate credit to the original author(s) and the source, provide a link to the Creative Commons license and indicate if changes were made.

The images or other third party material in this chapter are included in the chapter's Creative Commons license, unless indicated otherwise in a credit line to the material. If material is not included in the chapter's Creative Commons license and your intended use is not permitted by statutory regulation or exceeds the permitted use, you will need to obtain permission directly from the copyright holder. 